\title{
Non-anaemic iron deficiency should be investigated with the same priority as iron deficiency anaemia in fast track colorectal clinics-retrospective cohort study
}

\author{
Talal Majeed ${ }^{1}$, Joshua Solomon ${ }^{2}$, Romassa Siddiq Ali ${ }^{3}$, Praminthra Chitsabesan $^{4}$ \\ ${ }^{1}$ Department of Colorectal Surgery, ${ }^{2}$ Department of Upper GI Surgery, ${ }^{3}$ Department of Emergency Medicine, Wirral University Teaching Hospital, \\ Wirral, UK; ${ }^{4}$ Department of Coloproctology, York Hospital, York, UK \\ Contributions: (I) Conception and design: T Majeed, P Chitsabesan; (II) Administrative support: T Majeed, P Chitsabesan; (III) Provision of study \\ materials or patients: T Majeed, J Solomon, RS Ali; (IV) Collection and assembly of data: T Majeed, J Solomon, RS Ali; (V) Data analysis and \\ interpretation: T Majeed, J Solomon, RS Ali; (VI) Manuscript writing: All authors; (VII) Final approval of manuscript: All authors. \\ Correspondence to: Talal Majeed. Department of Colorectal Surgery, Wirral University Teaching Hospital, Wirral, UK. Email: talal.majeed@nhs.net.
}

Background: Since year 2000 the diagnostic criterion for fast track (FT) referrals for patients with
suspected colorectal cancer (CRC) is used in the UK. Iron deficiency anaemia (IDA) is one of the diagnostic
criteria. There is a strong evidence in the literature which suggests that Iron deficiency (ID) alone has a
strong relationship with CRC. Non-anaemic Iron deficiency (NAID) and all other types of anaemia are
investigated outside the scope of FT clinics. We postulated that patients with ID regardless of degree of
anaemia have an increased risk of CRC. By confirming this hypothesis, we can broaden the scope of the
diagnostic criterion for referral that can help to increase diagnostic yield of FT CRC services.
Methods: A retrospective observational cohort study was conducted from a dedicated data for FT clinics
from 2016-2018. Association between CRC and different forms of anaemia, Iron deficiency alone and bowel
symptoms was determined.

Results: Patients with iron deficiency (low MCV, MCH and ferritin) regardless of degree of anaemia were found more likely to have CRC. Factors like age, gender, family history and bowel symptoms (except abdominal mass) showed a very weak association with CRC in patients with ID.

Conclusions: ID without anaemia has a strong relationship with CRC and should be investigated with the same priority as IDA is investigated.

Keywords: Iron deficiency anaemia; iron deficiency; iron deficiency without anaemia; colorectal cancer (CRC); colorectal surgery; fast track clinics; two weeks wait; department of health diagnostic criterion

Submitted Dec 15, 2019. Accepted for publication Jun 18, 2020.

doi: 10.21037/jgo-19-451

View this article at: http://dx.doi.org/10.21037/jgo-19-451

\section{Introduction}

The Department of Health guidelines aimed at diagnosing colorectal cancer (CRC) at early curable stage. Diagnostic criterion was made to assist community physicians to identify high risk patients based on their symptoms and blood results. These criteria were based on recommendations from ACPGBI 2002 guidelines (1).

Patients with IDA with or without bowel symptoms can be referred to fast track CRC clinics, based on this criterion. Fast track service is a time pressured service and many patients miss the workup for ID before referral. As our data showed that $30 \%$ of patients had incomplete blood investigations. Also, some patients with established ID in the community can be missed FT investigations if they do not have anaemia.

Aim of this study to determine whether iron deficiency alone can predict presence of CRC in comparison to other 


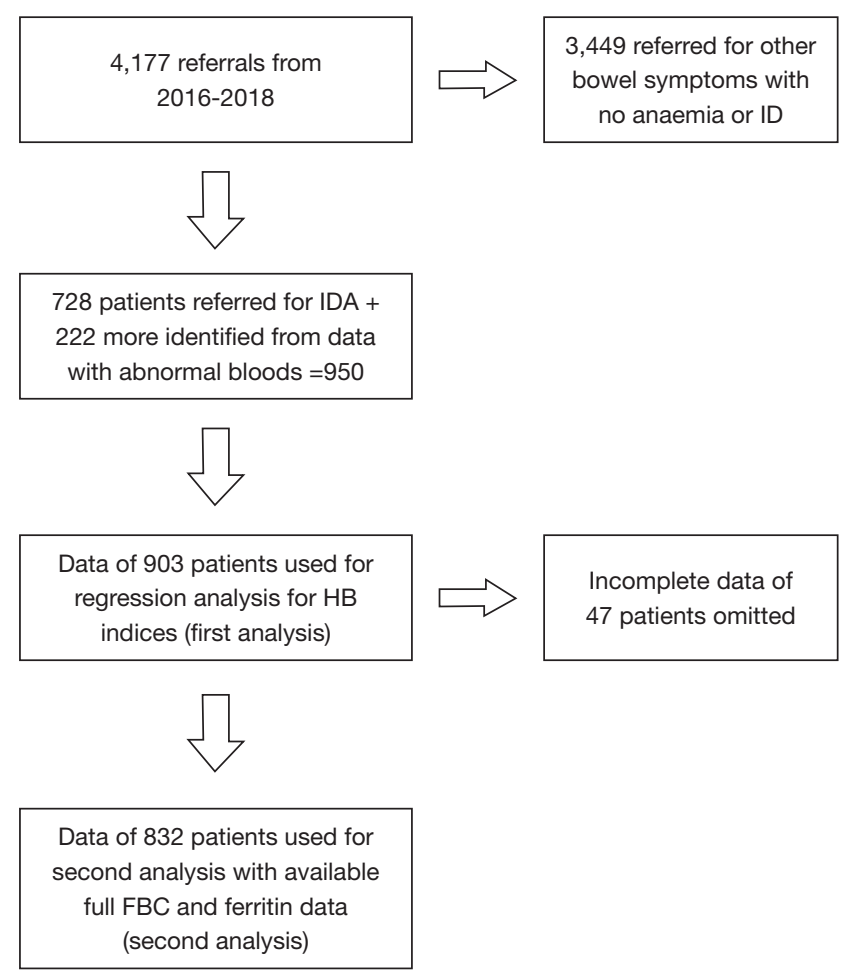

Figure 1 Flow diagram of data.

forms of anaemia and should be investigated in the same lines as IDA. We present the following article in accordance with the STROBE reporting checklist (available at http:// dx.doi.org/10.21037/jgo-19-451).

What this paper is adding: Latest BSG [2011] guidelines suggest presence of iron deficiency alone is enough to warrant bowel investigation (2). It is necessary to clear the concept of iron deficiency without anaemia.

If we determine that iron deficiency alone has strong association with CRC like IDA, it will change our practice of referring patients to fast track referral for CRC.

\section{Methods}

A retrospective observational cohort study was conducted in a busy district general hospital. Data was extracted from dedicated data base from April 2016-April 2018. In total 4,177 patients were referred to FT clinics. A total of 950 patients with abnormal blood tests were included in the study. Seven hundred twenty-eight patients were referred for IDA. While 222 patients were picked up from the data either had ID or any other type of anaemia. Patients with missing data $(n=47)$ were excluded from the study in the first analysis. Only 832 patient's data could be used in the second and final analysis (Figure 1). This work has been reported in line with STROCSS criteria to report cohort study. The article followed STROBE Checklist for reporting standards. Ethical approval obtained from local ethical committee and clinical governance. Data was anonymised according to guidelines set by NHS data protection policy.

\section{Inclusion and exclusion criterion}

Inclusion criterion:

(I) Patients referred for symptomatic or asymptomatic IDA;

(II) Patients identified from cohort who were referred for bowel symptoms but had Iron deficiency with normal haemoglobin and other types of anaemia.

Exclusion criterion:

Patients referred for bowel symptoms with normal blood (RBC indices and Iron studies).

Two types of data were analysed for an association with diagnosis of cancer. $T$-tests were used to compare those with and without a cancer diagnosis on red blood cell (RBC) indices, including haemoglobin (HB), mean corpuscular haemoglobin concentration (MCHC), mean corpuscular volume (MCV), mean corpuscular haemoglobin $(\mathrm{MCH})$, as well as serum ferritin levels. Levene's tests were used to assess differences in variance, and a $t$-test not assuming equal variances was used when the Levene's test was statistically significant. Pearson chi-square analyses were used to compare those with versus without a cancer diagnosis on the presence of various symptoms as well as on frequencies above and below the standard cut points for RBC indices and serum ferritin. Fisher's exact tests were used when expected cell frequencies were below five. A Mantel-Haenszel test was used to assess the trend in cancer prevalence across combined categories of serum ferritin and $\mathrm{MCV}$. An alpha of 0.05 was used as the level of significance (Table 1).

A second analysis was done to compare the yield of IDA, ID without anaemia and anaemia without ID with the help of McNemar's test.

Reference values:

Haemoglobin low cut-off value of $11 \mathrm{~g} / \mathrm{dL}$ for all men and $10 \mathrm{~g} / \mathrm{dL}$ for post-menopausal women were used as a reference (according to fast track referral guidelines).

MCV low cut-off value of 90 femtolitres per cell and for MCH 30 picograms was used as a low cut-off value (according to local lab guidelines). 
Table $1 \mathrm{RBC}$ indices and serum ferritin in patients with versus without a cancer diagnosis

\begin{tabular}{|c|c|c|c|c|c|c|c|c|c|}
\hline \multirow{2}{*}{ Blood indices } & \multicolumn{6}{|c|}{ Cancer diagnosis } & \multirow{2}{*}{$t$} & \multirow{2}{*}{$d f$} & \multirow{2}{*}{$\mathrm{P}$} \\
\hline & $\mathrm{N}$ & Mean & SD & $\mathrm{N}$ & Mean & SD & & & \\
\hline $\mathrm{MCHC}$ & 69 & 99.90 & 20.48 & 834 & 105.66 & 15.95 & 2.82 & 901 & 0.005 \\
\hline MCV & 69 & 79.25 & 8.23 & 830 & 82.50 & 8.70 & 3.00 & 897 & 0.003 \\
\hline Serum ferritin & 54 & 27.15 & 49.34 & 657 & 59.55 & 149.64 & $3.64^{*}$ & 156.2 & $<.001$ \\
\hline
\end{tabular}

*, $t$-test not assuming equal variances.

Table 2 Incidence of cancer by low versus normal RBC indices and serum ferritin

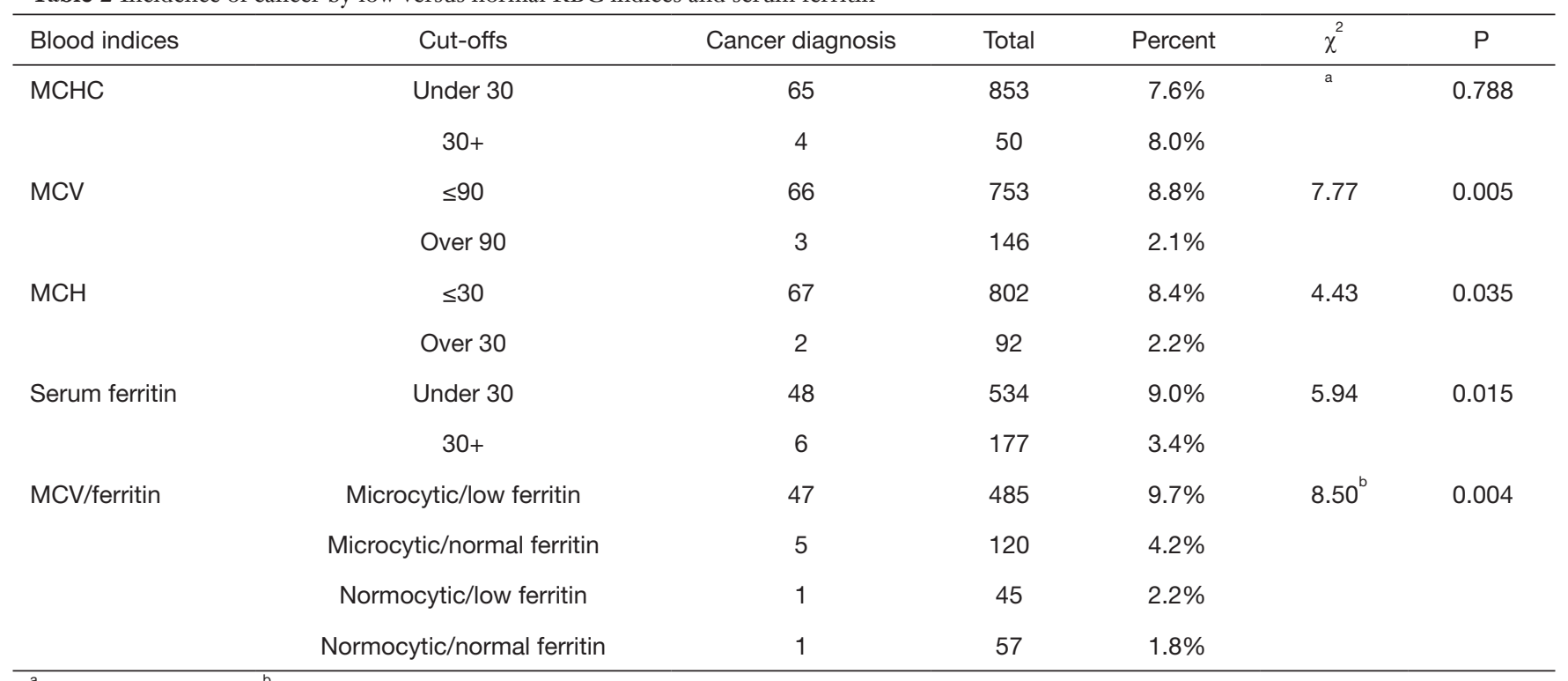

${ }^{\mathrm{a}}$, Fisher's exact test; ${ }^{\mathrm{b}}$, Mantel-Haenszel trend test.

\section{Results}

Male to female ratio was 49:51. Data was well distributed for range of age. Age ranged from 25-98 while median age was $75.6 \pm 10.4$. Seventy-three $(7.68 \%)$ patients were diagnosed with colorectal cancer.

\section{RBC indices}

The presence of a cancer diagnosis was assessed for with $\mathrm{RBC}$ indices and serum ferritin below the standard cutoffs. Patients with colorectal cancer had significantly low haemoglobin, mean cell volume, MCHC and serum ferritin. However, a multiple regression analysis shows that once haemoglobin is entered the regression as an only predictor of CRC, other values do not add significantly to the prediction beyond what can be explained by haemoglobin alone. This shows that haemoglobin and other factors (Iron deficiency depicted by MCV, MCHC and ferritin) assessed here are independent predictors of CRC. Table 2 presents the results. Except for MCHC, the incidence of cancer was significantly higher in patients with values below the cut-offs. The Mantel Haenszel trend test indicated that the incidence of cancer was highest in patients with a combination of low serum ferritin and low MCV (9.7\%), and decreased across categories, with only $1.8 \%$ of patients diagnosed with cancer when both $\mathrm{MCV}$ and serum ferritin were within normal ranges (Table 2).

The results of $t$-tests on the RBC indices and serum ferritin are presented in Table 1. As shown, mean levels of 
Table 3 Incidence of cancer by presence versus absence of symptoms

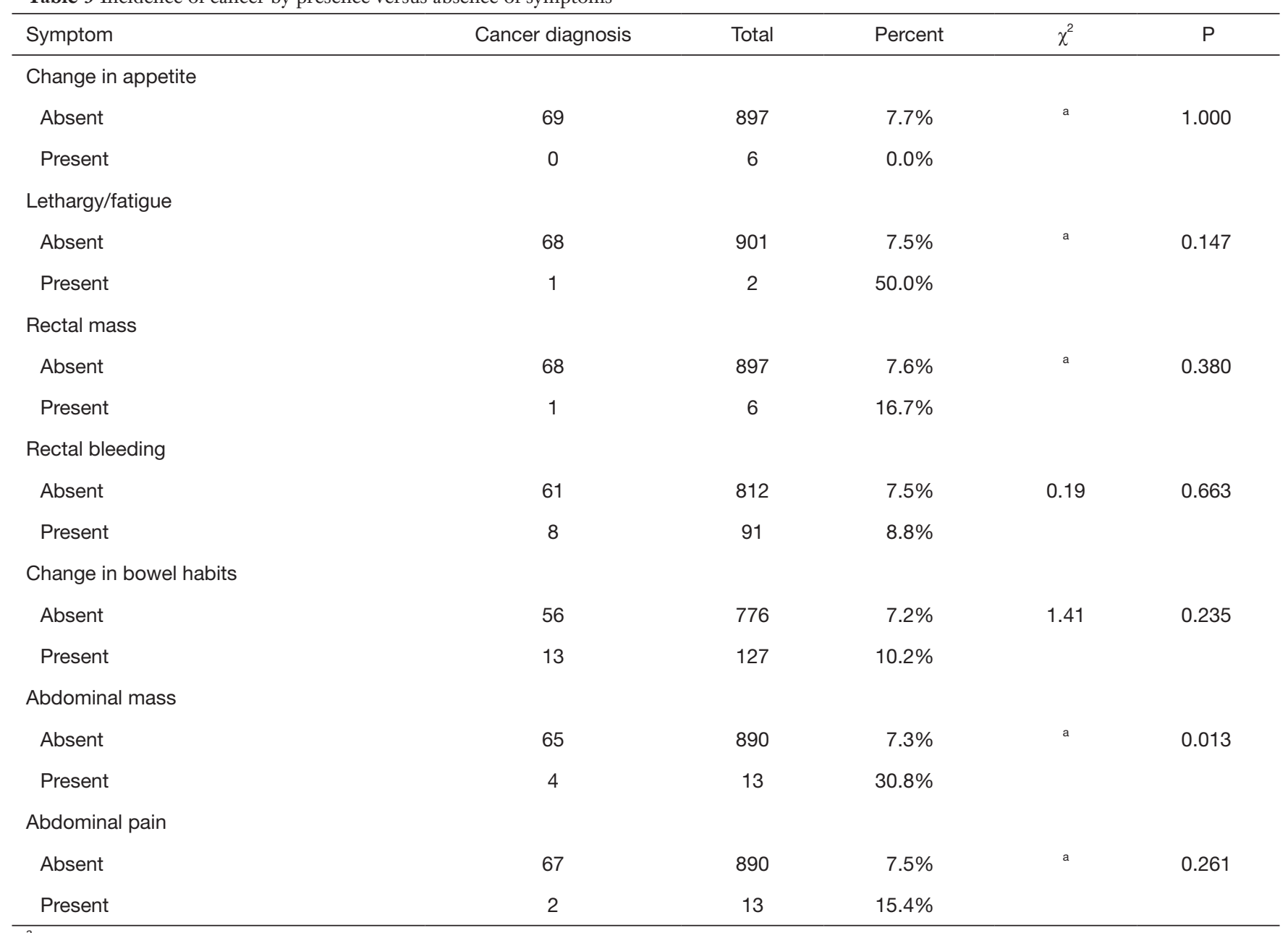

${ }^{\text {a }}$, Fisher's exact test.

all indices were significantly lower in those diagnosed with cancer.

\section{Symptomatology}

The presence of a cancer diagnosis was assessed in patients with a variety of symptoms in addition to their diagnosis of IDA. Table 3 presents the results. Of the seven symptoms reviewed, only the abdominal mass in the presence of iron deficiency was significantly related to a diagnosis of cancer (Table 3).

Gender, age and level of anaemia:

Overall diagnostic yield in our study was $7.47 \%$ with no statistically significant difference between symptomatic IDA and asymptomatic IDA.

MCV, MCHC and level of ferritin below cut-off were found to have strong association (alpha $<0.05)$ which was independent of levels of haemoglobin. While age, family history and gender were found to have a very week association with CRC.

Table 4 shows diagnostic yield and odds ratio comparison between IDA, ID with normal haemoglobin and Anaemia with no ID. Yield of CRC is significantly high in first two groups and relatively insignificant in patients who had anaemia with no ID. This proves that presence of iron deficiency has a strong predictive value of CRC.

\section{Discussion}

Bowel cancer is the 2nd most common cause of cancer death in the UK, accounting for $10 \%$ of all cancer deaths in 2017. Most of early stage bowel cancers are identified 
Table 4 Diagnostic yield and OR for IDA, ID and Anaemia without ID

\begin{tabular}{lcccc}
\hline ID vs. NAID vs. other anaemia & Cancer & No cancer & Yield & Odds ratio/confidence interval \\
\hline Low HB + low MCV low MCHC or low FER & 41 & 370 & $11.08 \%$ & $6.76(0.91-50.05)$ \\
Normal HB + low MCV low MCHC or low FER & 29 & 330 & $8.78 \%$ & $5.36(0.71-40.93)$ \\
Low HB + normal MCV or normal FER & 1 & 61 & $1.64 \%$ & $0.16(0.02-1.20)$ \\
\end{tabular}

by bowel screening program. Despite aiming at picking up almost $90 \%$ of bowel cancer cases, literature showed that only $24 \%$ of patients diagnosed with bowel cancer are referred through the FT route and still a large volume of patients are diagnosed through alternative routes (3). About $25 \%$ patients diagnosed with bowel cancer are diagnosed when they present in emergency (1). Patients who become symptomatic and seek emergency medical attention are usually at advanced stage of disease. There is a need to increase the performance of FT CRC as almost $90 \%$ of the health budget allocated for cancer diagnosis and screening is used on patients who do not have cancer (3). Our theory is based on the hypothesis that probably scope of guidelines for FT clinics is so narrow that we miss out several patients who could have CRC. In our study we have focussed on ID without anaemia and have tried to establish a strong relationship between ID alone with CRC.

In our study we have found that if patients have ID, then other factors are not very important. In other words, patients with unexplained ID should be investigated regardless of their age, gender and symptoms. Also, a common practice of judging the degree of anaemia based on levels of haemoglobin should be abandoned when ID is confirmed.

In our study we also explored the relationship between each blood index alone and in combination with ferritin levels to CRC. This make sense as mere blood indices (low $\mathrm{MCV}$ and $\mathrm{MCH}$ ) cannot define Iron deficiency and ferritin levels below the cut off must be used to define ID (4).

ID without anaemia is both diagnostic and clinical challenge as criterion to define it is not very clear and clinically it can go unnoticed for a long period of time. Waiting for haemoglobin to drop below a normal value in patients with iron deficiency can cause a delay in diagnosis. This can be explained by studying natural history of IDA (5). IDA starts with depletion of iron stores (gastrointestinal losses in case of malignancy). Iron depletion progresses to iron deficiency which can show up on blood picture as low ferritin, microcytosis and hypochromia. Low ferritin is usually first to appear on blood picture and is very sensitive to detect ID (3). Iron deficiency then advances to full blown iron deficiency anaemia. Iron deficiency anaemia is a late stage of iron deficiency takes time to develop and usually indicate long term iron deficiency. Natural history of IDA suggest that it should be investigated at early stage if the cause is malignancy. Literature does not suggest how long it takes for ID to develop into IDA but delaying investigations at this stage can upstage the tumour. Our study supports fast track referrals at ID stage but does not support confirmation of anaemia before referral (6).

Our study affirms the findings from 2011 guidelines for IDA from BSG. The guidelines suggest that red blood indices (mean cell volume, mean cell haemoglobin) and ferritin levels below normal cut-off range are specific indicators to diagnose iron deficiency. Hb levels are less specific and only a very low $\mathrm{Hb}$ indicates significant underlying pathology (3). Guidelines also suggest that Iron deficiency with normal $\mathrm{Hb}$ is very common presentation and should be investigated for bowel malignancy regardless of $\mathrm{Hb}$ levels (3). Despite these guidelines there was no change in diagnostic criterion for FT referrals until very recently. This could partially explain why FT CRC yield had always been poor.

Prior to 2017 the NHS National Institute for Health and Clinical Excellence referral guidelines for suspected lower GI cancer recommended that only patients with $\mathrm{Hb}$ concentration $<11 \mathrm{~g} / \mathrm{dL}$ in men or $<10 \mathrm{~g} / \mathrm{dL}$ in nonmenstruating women should be referred. With new recommendations it was accepted that these cut-off values can miss patients with colorectal cancer, particularly males. It is therefore recommended that any level of anaemia should be investigated in the presence of Iron deficiency. For the same reason NICE recommendations changed from a $\mathrm{Hb}$ cut off value to a more liberal definition of IDA to accommodate more patients at risk of CRC (7).

By not subjecting patients with ID without anaemia to FT investigations we can potentially miss patients with early stage CRC. Strict adherence to NICE guidelines can 
help to screen the population more holistically. Recent NICE guidelines can help to pick up more cancers and at an early stage. Our study is confirming the validity of these guidelines.

Based on our results we have recommended that both ID and IDA should be investigated for CRC. Our study recommendations are in line to core of BSG and NICE recommendations for management of IDA.

\section{Comparison with existing literature}

Sawhney, Mandeep et al. compared the prevalence of colonic neoplasia in anaemic patients with normal ferritin and low ferritin to those with low ferritin and no anaemia. In their study they showed ferritin alone (below a cut-off) is very sensitive in predicting CRC (8). They have also found that other laboratory parameters could not improve the predictive power of ferritin. Patient with normal ferritin were found to have the same likelihood of CRC as for patients with no anaemia. Their results corelate well with our study results and give further validity to latest NICE guidelines.

Ioannou and his colleagues in a population-based cohort looking for relationship between ID with no anaemia and gastrointestinal malignancy. They have found that patients with ID without anaemia have a five times greater chance of having CRC compared to patients with normal blood (9). They have advocated investigation of iron deficiency in the absence of anaemia, a condition that is far more prevalent than iron deficiency anaemia (6.4\% vs. 1.6\%).

Panagiotopoulou et al. have done a similar study have found in their study that microcytosis has a greater predictive value in diagnosing CRC when compare to anaemia or ferritin levels. They also observed that levels of haemoglobin were not statistically significantly linked to CRC (10). Their study attest to our findings to some extent as far as sensitivity of microcytosis for CRC is concerned. Although their study found no difference between yield of microcytic and Normocytic anaemia.

\section{Summary}

Our study results and literature review has showed that iron deficiency is as sensitive in detecting GI malignancy as IDA. Patients with normal haemoglobin with iron deficiency should be investigated with the same priority as patients with confirmed IDA.

\section{Strengths and limitations}

A limitation of our study is its retrospective nature and focussing only on patients referred to fast track clinics which constitutes only a quarter of all cancer referrals. We are studying a population who had already been selected for cancer referral and interplay of other confounding factors cannot be discounted. We were not able to prove that identifying and investigate patients with ID can help to detect cancer at early stage as data was small. Also, a small proportion of patients were referred without ferritin levels making it impossible to include these patients in the final analysis.

\section{Implications for research and/or practice}

These findings can become a basis for future referral guidelines and can help to improve the diagnostic yield of FT CRC clinics by ensuring that appropriate referrals are made to FT CRC clinics for anaemia. More research is needed by larger multi-institutional population-based cohort studies to explore this concept.

It also opens a possibility of establishing common anaemia pathway for patients with all other types of anaemia where they can be investigated by haematologists aided by gastroenterologists and colorectal surgeons thus laying off the workload from FT CRC clinics.

\section{Conclusions}

Confirmation of iron deficiency must be a pre-requisite before referring patients to FT CRC for anaemia. Patients with iron deficiency with normal haemoglobin should be investigated with the same priority as IDA.

\section{Acknowledgments}

Funding: None.

\section{Footnote}

Reporting Checklist: The authors have completed the STROBE reporting checklist. Available at http://dx.doi. org/10.21037/jgo-19-451

Data Sharing Statement: Available at http://dx.doi. org/10.21037/jgo-19-451 
Conflicts of Interest: All authors have completed the ICMJE uniform disclosure form (available at http://dx.doi. org/10.21037/jgo-19-451). The authors have no conflicts of interest to declare.

Ethical Statement: The authors are accountable for all aspects of the work in ensuring that questions related to the accuracy or integrity of any part of the work are appropriately investigated and resolved. The research was conducted in accordance with the Declaration of Helsinki (as revised in 2013). Ethical approval obtained from local ethical committee and clinical governance. Data was anonymised according to guidelines set by NHS data protection policy.

Open Access Statement: This is an Open Access article distributed in accordance with the Creative Commons Attribution-NonCommercial-NoDerivs 4.0 International License (CC BY-NC-ND 4.0), which permits the noncommercial replication and distribution of the article with the strict proviso that no changes or edits are made and the original work is properly cited (including links to both the formal publication through the relevant DOI and the license). See: https://creativecommons.org/licenses/by-nc-nd/4.0/.

\section{References}

1. Thorne K, Hutchings HA, Elwyn G. The effects of the Two-Week Rule on NHS colorectal cancer diagnostic

Cite this article as: Majeed T, Solomon J, Ali RS, Chitsabesan P. Non-anaemic iron deficiency should be investigated with the same priority as iron deficiency anaemia in fast track colorectal clinics-retrospective cohort study. J Gastrointest Oncol 2020;11(4):609-615. doi: 10.21037/jgo-19-451 services: a systematic literature review. BMC Health Serv Res 2006;6:43.

2. Georgiou A, Khakoo S, Edwards P, et al. Outcomes of Patients with Early Onset Colorectal Cancer Treated in a UK Specialist Cancer Center. Cancers (Basel) 2019;11:1558.

3. Goddard AF, James MW, McIntyre AS, et al. Guidelines for the management of iron deficiency anaemia. Gut 2011;60:1309-16.

4. Camaschella C. Iron-deficiency anemia. N Engl J Med 2015;372:1832-43.

5. Soppi ET. Iron deficiency without anemia - a clinical challenge. Clin Case Rep 2018;6:1082-6.

6. Dickey S, Rockey DC. The Natural History of Iron Deficiency Anemia. Am J Med Sci 2019;358:357-62.

7. Benton S, Steele R, Logan R, et al. NICE referral guidelines for suspected cancer: colorectal cancer and faecal occult blood testing. Ann Clin Biochem 2016;53:7-9.

8. Sawhney MS, Lipato T, Nelson DB, et al. Should patients with anemia and low normal or normal serum ferritin undergo colonoscopy? Am J Gastroenterol 2007;102:82-8.

9. Ioannou GN, Rockey DC, Bryson CL, et al. Iron deficiency and gastrointestinal malignancy: a populationbased cohort study. Am J Med 2002;113:276-80.

10. Panagiotopoulou IG, Fitzrol D, Parker RA, et al. The yield of colorectal cancer among fast track patients with normocytic and microcytic anaemia. Ann R Coll Surg Engl 2014;96:289-93. 